\title{
Oleanolic acid protects against diabetic cardiomyopathy via modulation of the nuclear factor erythroid 2 and insulin signaling pathways
}

\author{
WEI-FANG LI ${ }^{1}$, PENG WANG ${ }^{2}$, HUA LI ${ }^{1}$, TIAN-YI LI ${ }^{1}$, MING FENG $^{1}$ and SU-FANG CHEN ${ }^{1}$ \\ ${ }^{1}$ Department of Geriatric Endocrinology, The First Affiliated Hospital of Zhengzhou University, Zhengzhou, Henan 450052; \\ ${ }^{2}$ Department of Epidemiology, College of Public Health, Zhengzhou University, Zhengzhou, Henan 450001, P.R. China
}

Received December 28, 2015; Accepted January 20, 2017

DOI: $10.3892 / \mathrm{etm} .2017 .4527$

\begin{abstract}
Oleanolic acid (OL) is a pentacyclic triterpene compound used for the treatment of hepatitis, liver fibrosis and liver cirrhosis. In China, there is no published research on the effect or biological utilization of OL on liver diseases. The aim of the present study was to investigate the protective effects of OL against diabetic cardiomyopathy and its possible mechanism. A rat model of diabetes was established using streptozotocin and the effect of OL on diabetic cardiomyopathy (DCM) was evaluated. The results demonstrated that OL significantly reversed the DCM-induced changes to body weight, heart rate, echocardiography and hemodynamics, phosphorylated-glycogen synthase (GS) and glycogen phosphorylase (GP) activity in diabetic rats (all $\mathrm{P}<0.01$ ). Treatment of diabetic rats with OL significantly inhibited oxidative stress and activated heme oxygenase (HO)-1/nuclear factor erythroid 2 (Nrf2) signaling in a rat model of diabetes (both $\mathrm{P}<0.01$ ). The results of the present study indicate that OL protects against DCM through the HO-1/Nrf2 and insulin modulating GS/GP signaling pathways.
\end{abstract}

\section{Introduction}

In recent years, the incidence rates of diabetes have been increasing each year (1). Currently, diabetes is the fifth leading cause of mortality following infectious diseases, cardiovascular diseases, cancer and trauma (1). Diabetes is a chronic progressive disease characterized by high blood glucose levels. There are two types of diabetes, Type I and Type II, which are characterized by the cause of the disease (2). Type II diabetes is associated with a high fat diet and obesity, is characterized

Correspondence to: Professor Wei-Fang Li, Department of Geriatric Endocrinology, The First Affiliated Hospital of Zhengzhou University, 1 Jianshe Road, Zhengzhou, Henan 450052, P.R. China E-mail: queganhepo1262318@126.com

Key words: oleanolic acid, diabetic cardiomyopathy, nuclear factor erythroid 2, insulin by insulin resistance and accounts for $\sim 90 \%$ of cases of adult diabetes (3). Type II diabetes is responsible for $\sim 5.2 \%$ of the global mortality rate (4).

Diabetes may cause systemic microvascular and large vascular lesions, diabetic retinopathy, diabetic nephropathy, diabetic cerebrovascular disease and diabetic cardiovascular disease (5). In addition to causing vascular disease, diabetes may cause myocardial cell damage and eventually lead to the development of diabetic cardiomyopathy (DCM) (6). DCM is a disease caused by diabetes that is independent of coronary artery disease, hypertension and heart valve disease. The main characteristics of DCM include oxidative stress, cardiac hypertrophy, apoptosis, myocardial fibrosis and impaired cardiac function (7).

Nuclear factor erythroid 2 (Nrf2), one of the 'cap 'n' collar' family members, is a master regulator of cell toxicity and redox (8). When cells are affected by oxidative stress or electrophilic compound stimulation, $\mathrm{Nrf} 2$ will translocate into the nucleus and bind to an antioxidant response element to induce the expression of genes required for the antioxidant defense system (9). The antioxidant defense system is the main protective mechanism against oxidative damage, resulting in the neutralization of oxidants and electrophiles by antioxidants in the cells (10).

Oleanolic acid (OL) is a pentacyclic triterpenoid [chemical name, (3P)-3-hydroxy-olean-12-en-28-oic acid] with a natural chemical composition formed from free or combined glycosides. OL widely exists in plants, such as white snake tongue grass, hawthorn fruit, clove, papaya, Ligustrum lucidum and Prunella vulgaris (11). Experimental studies have demonstrated that OL has effective liver and kidney protective properties, inhibits platelet aggregation and has hypolipidemic, hypoglycemic, anti-inflammatory, anti-cancer, anti-stress, anti-ulcer and anti-microbial properties $(12,13)$. OL is used to treat acute jaundice, chronic poisoning hepatitis, liver fibrosis and cirrhosis (12). In addition, OL is effective in the recovery of immune function caused by chemotherapy for malignant tumors and protection against liver damage following administration anti-tuberculosis agents (14). Therefore, the aim of the present study was to investigate the effects of OL against DCM in a rat model of diabetes and the possible mechanism. 


\section{Materials and methods}

Animals and induction of DCM in rats. The experimental procedures were approved by the Animal Care and Welfare Committee of The First Affiliated Hospital of Zhengzhou University (Zhengzhou, China) in accordance with the guidelines for the Care and Use of Laboratory Animals. A total of 24 male Sprague-Dawley rats (220-250 g; 6-8 weeks old) were purchased from the Experimental Animal Center of the Academy of The First Affiliated Hospital of Zhengzhou University and maintained in standard conditions at $22 \pm 2^{\circ} \mathrm{C}$, $55 \pm 5 \%$ humidity with a $12 \mathrm{~h}$ light/dark cycle and fed normal chow. All rats were randomly assigned into three groups: Control group $(n=8)$, DCM model group $(n=8)$ and OL treatment group $(n=8)$. Diabetes was induced in the DCM model group using an intraperitoneal injection of streptozotocin (STZ; 60 mg/kg; Sigma-Aldrich; Merck Millipore, Darmstadt, Germanu). The OL treatment group were administered with an intraperitoneal injection of $80 \mathrm{mg} / \mathrm{kg}$ OL once every 2 days for 14 days. The control group was administered equal volumes of normal saline.

Body weight and heart rate. A CODA 8-channel tailcuff blood pressure system (Kent Scientific, Torrington, CT, USA) was used to record heart rate. Subsequent to sacrifice, body weights were immediately measured. Rats were washed with phosphate-buffered saline and their wet weight was recorded using standard scales.

Echocardiography and hemodynamic measurements. Left ventricular internal dimension in systole (LVIDs), left ventricular internal dimension in diastole (LVIDd), interventricular septal wall thickness (IVSd) and fractional shortening were measured using a manometer-tipped catheter (SPR-320NR; Millar, Inc., Houston, TX, USA) and recorded using a MP150 system (Biopac Systems, Goleta, CA, USA).

Determination of p-glycogen synthase (GS), glycogen phosphorylase $(G P)$, methane dicarboxylic aldehyde (MDA) and superoxide dismutase (SOD) activities. The GS, GP, MDA (A003-1) and SOD (A001-3; both Nanjing Jiancheng Bioengineering Institute, Nanjing, China) activities were assayed radiochemically using ELISA kits, according to the manufacturer's instructions. The GS and GP activities were expressed as $\mathrm{nmol} / \mathrm{min} / \mathrm{mg}$ protein, MDA activity was expressed as nmol/mg protein and SOD activity was expressed as U/mg protein using an ultraviolet-visible spectrophotometer (UVmini-1240; Shimadzu Corp., Kyoto, Japan).

Determination of glycogen content. Heart tissue samples were boiled in $30 \% \mathrm{KOH}$ saturated with $\mathrm{NaSO}_{4}$ for $30 \mathrm{~min}$. Glycogen content was precipitated with $95 \%$ ice-cold ethanol for $30 \mathrm{~min}$. Following this, $5 \mathrm{ml}$ sulfuric acid was added and the mixture was incubated on ice for $30 \mathrm{~min}$. Glycogen content was measured using an ultraviolet-visible spectrophotometer (UVmini-1240; Shimadzu Corp.) at $490 \mathrm{~nm}$.

Western blot analysis. Rat hearts $(50 \mathrm{mg})$ were homogenized in radioimmunoprecipitation assay buffer (Beyotime Institute of Biotechnology, Haimen, China) supplemented with a

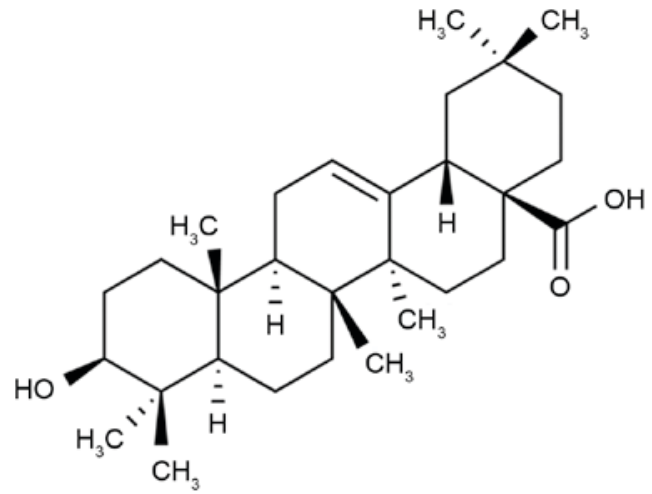

Figure 1. Chemical structure of oleanolic acid.

protease inhibitor cocktail. Total protein concentrations were measured using a BCA protein assay kit (Pierce; Thermo Fisher Scientific, Inc., Waltham, MA, USA). A total of $50 \mu \mathrm{g}$ protein from each sample was separated by $8-10 \%$ SDS-PAGE and transferred onto polyvinylidene difluoride membranes (Merck Millipore). Membranes were blocked with 5\%-skim milk powder in TBS with Tween-20 (TBST) and incubated with anti-heme oxygenase (HO)-1 (1:200; sc-10789), anti-Nrf2 (1:300; sc-33569), anti-phosphorylated-GP (p-GP; 1:200; sc-66913), anti-phosphorylated GS (p-GS; 1:400; sc-99029) and anti- $\beta$-actin (1:500; sc-130656; all Santa Cruz Biotechnology, Inc., Dallas, TX, USA) primary antibodies at $4^{\circ} \mathrm{C}$ overnight. Following washing three times with TBST, the membranes were incubated with a secondary horseradish peroxidase-conjugated immunoglobulin $\mathrm{G}$ antibody $(1: 3,000$; sc-2357; Santa Cruz Biotechnology, Inc.) at $37^{\circ} \mathrm{C}$ for $1 \mathrm{~h}$, visualized using an enhanced chemiluminescence reagent kit (P0018A; Beyotime Institute of Biotechnology) and quantified using Image J 3.0 software (National Institutes of Health, Bethesda, MD, USA).

Statistical analysis. All data are expressed as the mean \pm standard error of the mean using InStat version 5 (GraphPad Software, Inc., La Jolla, CA, USA). Data between two groups were compared using unpaired t-tests. Comparisons for three or more groups were performed using one-way or two-way analysis of variance. $\mathrm{P}<0.05$ was considered to indicate a statistically significant difference.

\section{Results}

OL protects against body weight and heart rate alterations in rats with DCM. Fig. 1 presents the chemical structure of OL. The body weights of rats with DCM were lower than those of the control rats following treatment $(\mathrm{P}=0.0081$; Fig. 2A) and the heart rates of rats with DCM were higher than those of the control rats $(\mathrm{P}=0.0042$; Fig. 2B). Treatment with OL significantly increased the DCM-induced reduction of body weight $(\mathrm{P}=0.0096)$ and significantly decreased the DCM-induced increase in heart rate $(\mathrm{P}=0.0067)$ in rats with DCM (Fig. 2).

OL protects against alterations to echocardiography in rats with DCM. To investigate whether OL protects against 
A

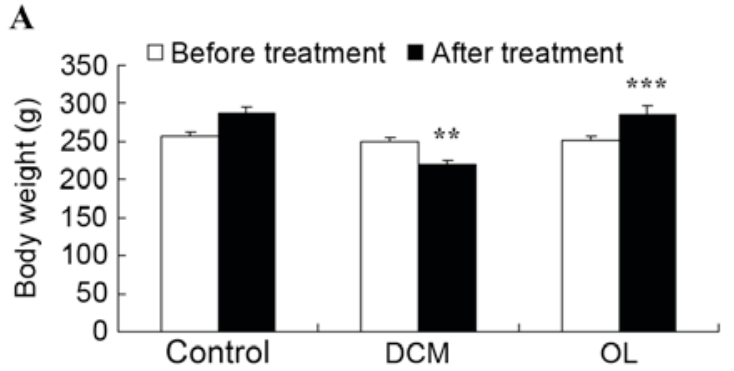

B

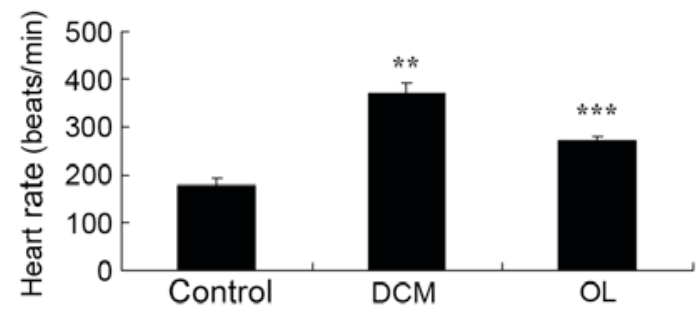

Figure 2. Effects of OL on body weight and heart rate in rats with DCM. (A) OL treatment protects against decreases in body weight of rats with DCM. (B) OL treatment protects against increases in the heart rates of rats with DCM. Data are presented as mean \pm standard error of the mean. ${ }^{* * *} \mathrm{P}<0.01 \mathrm{vs}$. the control group; ${ }^{* * *} \mathrm{P}<0.01$ vs. the DCM model group. OL, oleanolic acid; DCM, diabetic cardiomyopathy.

A

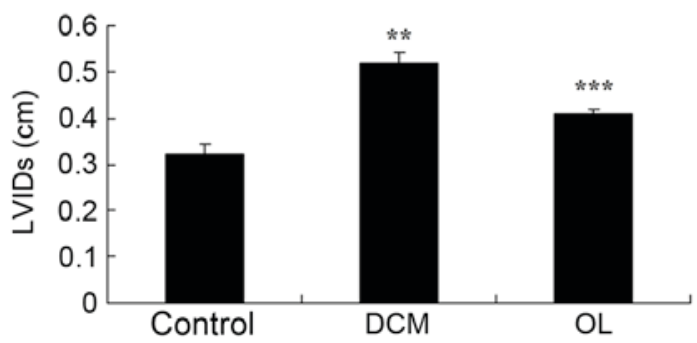

B

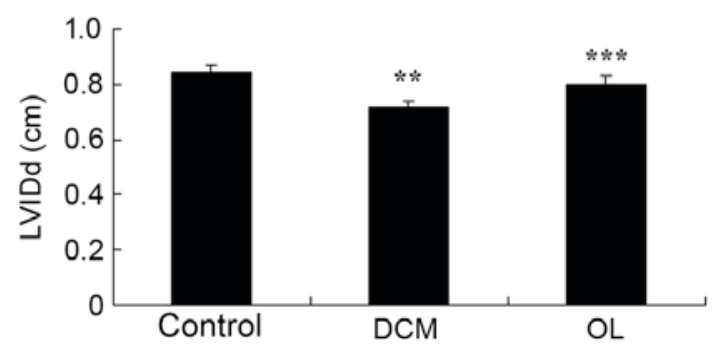

Figure 3. Effects of OL on echocardiography in rats with DCM. (A) OL decreased DCM-induced increases in LVIDs in rats with DCM. (B) OL increased DCM-induced decreases in LVIDd in rats with DCM. Data are presented as mean \pm standard error of the mean. ${ }^{* * *} \mathrm{P}<0.01$ vs. the control group; ${ }^{* * * *} \mathrm{P}<0.01 \mathrm{vs.}$. the DCM model group. OL, oleanolic acid; DCM, diabetic cardiomyopathy; LVIDs, left ventricular internal dimension in systole; LVIDd, left ventricular internal dimension in diastole.

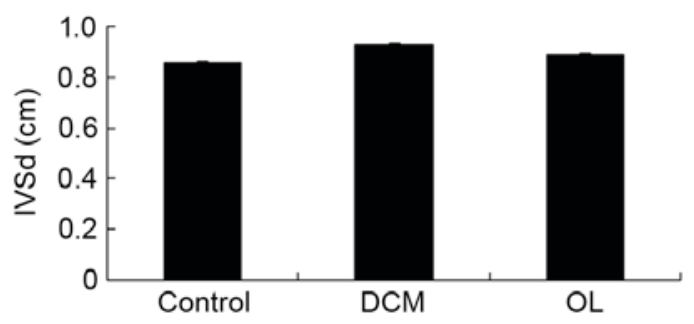

B

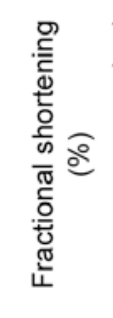

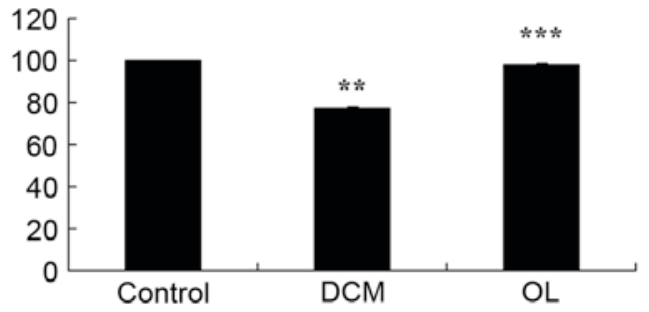

Figure 4. Effects of OL on the hemodynamics of rats with DCM. (A) No significant differences were observed between the IVSd measurements of the control, DCM and OL treatment groups; however, (B) OL-treatment increased the fractional shortening in rats with DCM compared with the untreated rats with DCM Data are presented as mean \pm standard error of the mean. ${ }^{* *} \mathrm{P}<0.01$ vs. the control group; ${ }^{* * *} \mathrm{P}<0.01$ vs. the DCM model group. OL, oleanolic acid; DCM, diabetic cardiomyopathy; IVSd, interventricular septal wall thickness.

echocardiographic alterations in rats with DCM, LVIDs and LVIDd were measured. LVIDs and LVIDd were significantly higher $(\mathrm{P}=0.0063)$ and lower $(\mathrm{P}=0.0086)$, respectively, in rats with DCM compared with normal controls (Fig. 3). Treatment with OL significantly inhibited decreased $(\mathrm{P}=0.0077)$ and increased ( $\mathrm{P}=0.0096)$ LVIDs and LVIDd measurements, respectively, as compared with rats with DCM (Fig. 3).

OL protects against alterations in hemodynamics in rats with DCM. Fig. 4 demonstrates the effects of OL on the hemodynamics of rats with DCM. No significant differences were observed in IVSd between the three groups; however, rats with DCM exhibited a significant decrease in fractional shortening $(\mathrm{P}=0.0067)$ compared with the control rats. Treatment with OL decreased the DCM-induced reduction of fractional shortening, resulting in a significant increase $(\mathrm{P}=0.0073)$ in fractional shortening in the OL treatment group compared with the DCM model group (Fig. 4).

OL protects against increased glycogen levels in rats with $D C M$. The present study demonstrated that DCM significantly increased blood glycogen levels in rats with DCM $(\mathrm{P}=0.0031)$ compared with the control group; however, treatment with OL significantly reduced $(\mathrm{P}=0.0068)$ the blood glycogen levels of the rats in the treatment group compared with the glycogen levels of the untreated rats with DCM (Fig. 5).

OL protects against oxidative stress in rats with DCM. In the present study, rats with DCM exhibited a significant increase $(\mathrm{P}=0.0022)$ in MDA activity and a significant decrease 


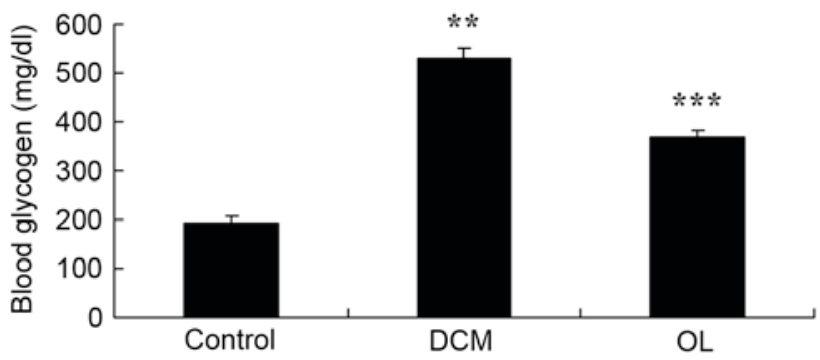

Figure 5. Effect of OL on blood glycogen levels in rats with DCM. OL-treatment decreased the blood glycogen level in rats with DCM compared with the untreated rats with DCM. Data are presented as mean \pm standard error of the mean. ${ }^{* *} \mathrm{P}<0.01$ vs. the control group; ${ }^{* * *} \mathrm{P}<0.01$ vs. the $\mathrm{DCM}$ model group. OL, oleanolic acid; DCM, diabetic cardiomyopathy.

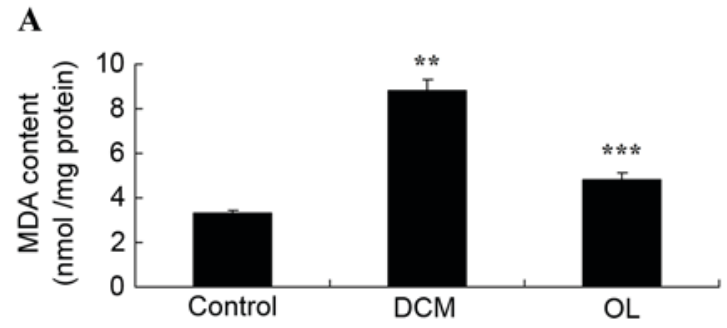

B

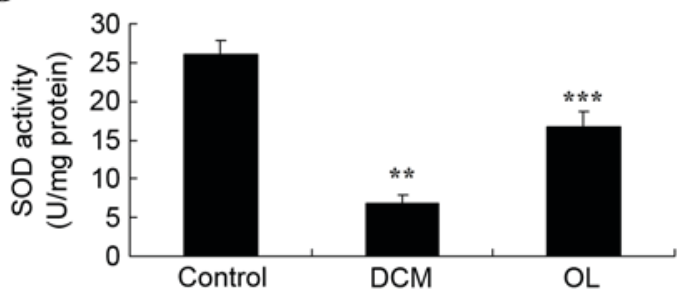

Figure 6. Effects of OL on oxidative stress in rats with DCM. (A) OL protects against increased MDA activity in rats with DCM. (B) OL increased SOD activity in rats with DCM. Data are presented as mean \pm standard error of the mean. ${ }^{* *} \mathrm{P}<0.01$ vs. the control group; ${ }^{* * *} \mathrm{P}<0.01$ vs. the DCM model group. OL, oleanolic acid; DCM, diabetic cardiomyopathy; MDA, methane dicarboxylic aldehyde; SOD, superoxide dismutase.

$(\mathrm{P}=0.0013)$ in SOD activity compared with control group rats (Fig. 6). Rats treated with OL exhibited decreased oxidative stress through the significant suppression $(\mathrm{P}=0.0052)$ of MDA activity and the significantly increased $(\mathrm{P}=0.0038)$ levels of SOD activity compared with untreated rats with DCM (Fig. 6).

OL protects against decreased $\mathrm{HO}-1$ protein expression levels in rats with DCM. In order to elucidate the possible roles of the HO-1 protein, the present study determined HO-1 protein expression levels in vivo. Results demonstrated that there was a significant decrease $(\mathrm{P}=0.0031)$ in the $\mathrm{HO}-1$ protein expression level in rats with DCM when compared with the control group; however, OL-treated rats exhibited significantly increased $(\mathrm{P}=0.0067) \mathrm{HO}-1$ protein expression levels compared with untreated rats with DCM (Fig. 7).

OL protects against decreased Nrf2 protein expression levels in rats with DCM. In order to elucidate the role of $\mathrm{Nrf} 2$ and the effects of OL against DCM, the present study measured Nrf2 protein expression levels using western blot analysis. As

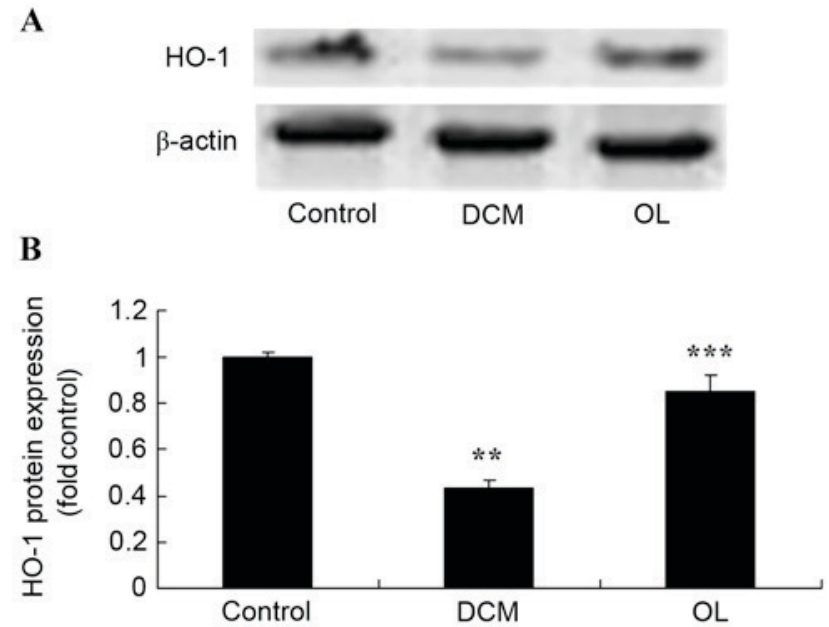

Figure 7. Effect of OL on HO-1 protein expression levels in rats with DCM. (A) OL treatment increased the HO-1 protein expression level in rats with DCM according to western blot analysis. (B) Quantitative analysis of HO-1 protein expression level following OL treatment in rats with DCM. Data are presented as mean + standard error of the mean. ${ }^{* *} \mathrm{P}<0.01$ vs. the control group; ${ }^{* * *} \mathrm{P}<0.01$ vs. the DCM model group. OL, oleanolic acid; DCM, diabetic cardiomyopathy; HO-1, heme oxygenase 1.

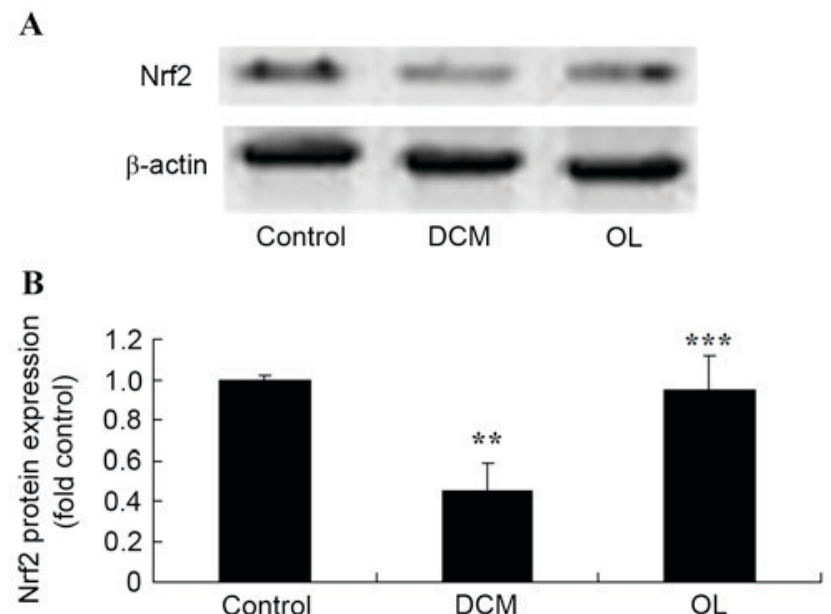

Figure 8. Effects of OL on Nrf2 protein expression levels in rats with DCM. (A) OL treatment increased the Nrf2 protein expression level in rats with DCM according to western blot analysis. (B) Quantitative analysis of Nrf2 protein expression level following OL treatment in rats with DCM. Data are presented as mean \pm standard error of the mean. ${ }^{* *} \mathrm{P}<0.01$ vs. the control group; ${ }^{* * *} \mathrm{P}<0.01$ vs. the DCM model group. OL, oleanolic acid; DCM, diabetic cardiomyopathy; Nrf2, nuclear factor erythroid 2.

shown in Fig. 8, DCM significantly inhibited $(\mathrm{P}=0.0046)$ the protein expression levels of $\mathrm{Nrf} 2$ compared with control rats; however, treatment with OL significantly increased $(\mathrm{P}=0.0072)$ the Nrf2 protein expression levels in DCM rats compared with the untreated DCM model group.

$O L$ protects against increased GS activity and protein expression levels in rats with DCM. In order to determine the effects of OL on the GS signaling pathway, the activity and protein expression levels of GS were measured using an ELISA kit and western blot analysis. Results demonstrated that the activity and protein expression levels of GS were significantly increased $(\mathrm{P}=0.0061)$ in rats with $\mathrm{DCM}$ compared with the 
A

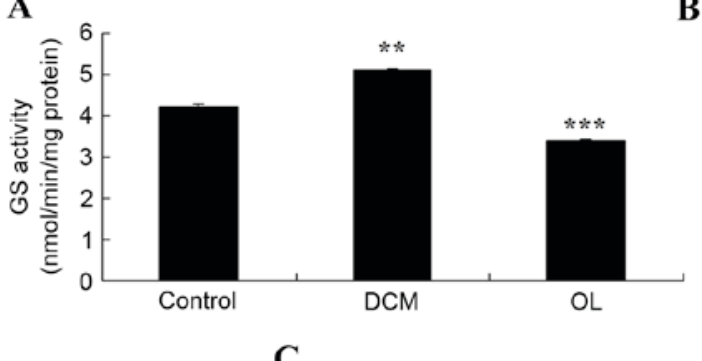

C
B

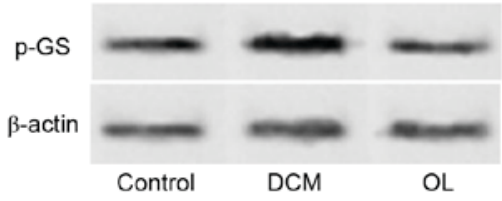

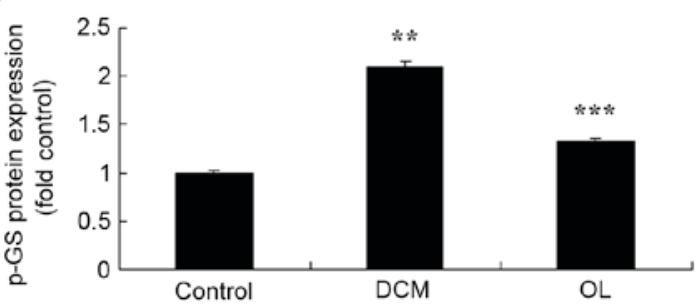

Figure 9. Effects of OL on GS activity and protein expression levels in rats with DCM. (A) OL treatment decreased the GS activity in rats with DCM, as determined by ELISA. (B) Western blotting demonstrated that OL treatment decreased p-GS protein expression levels in rats with DCM. (C) Quantitative analysis of the p-GS protein expression levels in rats with DCM. Data are presented as mean \pm standard error of the mean. ${ }^{* *} \mathrm{P}<0.01$ vs. the control group;

${ }^{* * *} \mathrm{P}<0.01$ vs. the DCM model group. OL, oleanolic acid; DCM, diabetic cardiomyopathy; GS, glycogen synthase; p, phosphorylated.

A

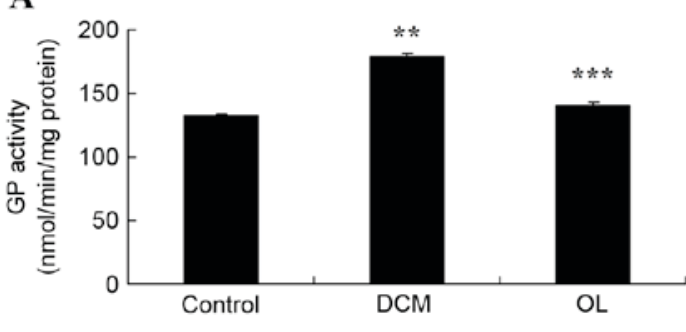

B

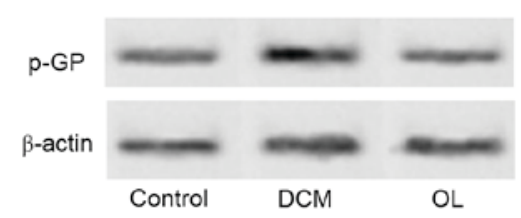

C

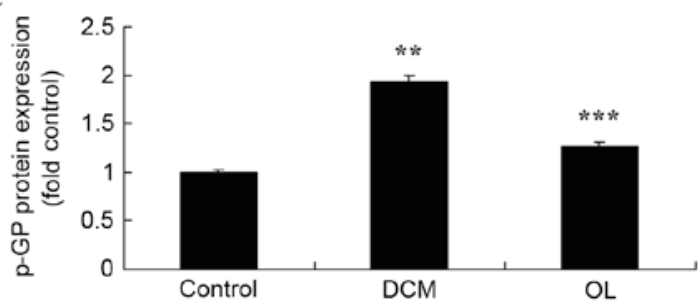

Figure 10. Effects of OL on GP activity and protein expression levels in rats with DCM. (A) OL treatment decreased the GP activity in rats with DCM, as determined by ELISA. (B) Western blotting demonstrated that OL treatment decreased p-GP protein expression levels in rats with DCM. (C) Quantitative analysis of the p-GP protein expression levels in rats with DCM. Data are presented as mean \pm standard error of the mean. ${ }^{* *} \mathrm{P}<0.01 \mathrm{vs.}$ the control group; ${ }^{* * *} \mathrm{P}<0.01$ vs. the DCM model group. OL, oleanolic acid; DCM, diabetic cardiomyopathy; GP, glycogen phosphorylase; p, phosphorylated.

control group (Fig. 9). Treatment with OL significantly inhibited $(\mathrm{P}=0.0078) \mathrm{GS}$ activity and protein expression levels in rats with DCM compared with the untreated DCM model group (Fig. 9).

OL protects against increased GP activity and protein expression levels in rats with DCM. In order to determine whether OL affects the GP signaling pathway in order to protect against DCM in rats, GP activity and protein expression levels were measured using an ELISA kit and western blot analysis. Results demonstrated that the activity and protein expression levels of GP were significantly increased $(\mathrm{P}=0.0066)$ in rats with DCM compared with the control group (Fig. 10). Treatment with OL significantly inhibited $(\mathrm{P}=0.0081) \mathrm{GP}$ activity and protein expression levels in rats with DCM compared with the untreated DCM model group (Fig. 10).

\section{Discussion}

DCM is a cardiac disease that is distinct from hypertension and coronary artery atherosclerotic heart disease (1). Research has confirmed the existence of DCM and it is believed that a particularly significant symptom of the disease is left ventricular diastolic dysfunction. As the disease progresses, symptom develop into left ventricular hypertrophy and systolic dysfunction occurs and, ultimately, this may result in the development of heart failure (7). In the present study, it was demonstrated that OL protects against 
DCM-induced body weight and heart rate alterations and improves echocardiographic and hemodynamic measurements in DCM rats.

Currently, the specific pathogenesis of DCM is unclear and, due to the lack of clinical research on this disease, correct diagnosis and treatment of DCM is often difficult, with the disease often being misdiagnosed as coronary heart disease. In the majority of cases, the diagnosis of DCM is confirmed by autopsy (15). Previous studies have demonstrated that the occurrence and development of DCM is related to a variety of factors $(16,17)$. Due to disruption of the cellular antioxidant defense balance in DCM, increased numbers of free radicals are generated, resulting in oxidative stress and cell damage. Possible mechanisms for this damage include metabolic disorders, changes in tissue structure and function, small vessel disease, autonomic dysfunction, insulin resistance and cell factor abnormalities (9). In the present study, OL treatment inhibited the increased glycogen content in the blood of rats with DCM. A study by Mukundwa et al (14) demonstrated that OL affects the insulin signaling pathway of diabetic male Sprague-Dawley rats.

A previous study has demonstrated that oxidative stress is the most important factor in DCM development (18). Oxidative stress results in the increase of intracellular oxidative metabolites and, when cell antioxidant mechanisms are lacking, it promotes the accumulation of reactive oxygen species, resulting in cell toxicity (19). Oxidative stress may result from various causes, including myocardial cell damage, exercise, psychological stress, ischemia and hypoxia $(17,20)$. Recent studies have demonstrated that, when using different blood glucose gradients to develop myocardial cells, cell apoptosis is proportional to concentration of blood glucose, with high blood glucose levels inducing oxidative stress through the generation of reactive oxygen species $(21,22)$. The increased number of reactive oxygen species results in gene expression abnormalities, alterations to signal transduction and activation of the programmed cell death pathway $(23,24)$. Inhibition of myocardial cell death can significantly prevent heart toxicity in diabetes $(23,24)$. In the present study, it was observed that OL reduced oxidative stress in rats with DCM. A study by Sarkar et al (23) demonstrated that OL prevents fluoride-induced metabolic and oxidative stress in rat brain through the suppression of oxidative stress.

$\mathrm{HO}-\mathrm{l}$ is a microsomal oxidase with various functions in the process of heme metabolism (10). HO-1 is a myocardial protection factor and has anti-inflammatory, anti-oxidant, anti-apoptotic and anti-arrhythmia properties. In addition, HO-1 has an important role in cardiovascular disease and research has demonstrated that $\mathrm{HO}-1$ is able to inhibit cardiac hypertrophy; however, oxidative stress leads to DCM and results in the reduction of HO-1 expression $(24,25)$. HO-1 expression has become a universal marker of protection against oxidative stress. In the present study, treatment of the STZ-induced rat model of DCM with OL significantly activated HO-1 protein expression in rats with DCM. A study by Hong et al (26) suggested that OL attenuates tubulointerstitial fibrosis through $\mathrm{Nrf} 2 / \mathrm{HO}-1$ signaling in chronic cyclosporine nephropathy.

Studies have demonstrated that Nrf2 protection against heart damage due to oxidative stress may involve various mechanisms, including: Regulation of the sensitivity of apoptosis by Nrf2; and, Nrf2 inhibiting the Fas-induced apoptosis pathway, thus, Nrf2 overexpression may protect cells against Fas-induced apoptosis $(8,27)$. Nrf2 has anti-inflammatory effects, inhibiting foam cell formation and pathogenesis formation of atherosclerosis (28). In the present study, treatment with OL significantly increased Nrf2 protein expression levels and significantly suppressed the protein expression levels and activity of GS and GP in rats with DCM. A study by Liu et al (11) reported that OL alleviates ethanol-induced hepatic injury through $\mathrm{Nrf} 2$ in rats.

In conclusion, the present study identified novel protective effects of OL against DCM through the inhibition of DCM-induced body weight and heart rate alterations, improved echocardiographic and hemodynamic measurements, and reduced blood glycogen levels in DCM rats. These findings demonstrate that the $\mathrm{HO}-1 / \mathrm{Nrf} 2$ signaling pathway has an important role in OL protection against cardiac damage through insulin modulation of the GS/GP signaling pathway in rats with DCM. These findings also indicate that OL may have the potential to be used as a novel therapeutic treatment for DCM.

\section{References}

1. Saengtipbovorn S and Taneepanichskul S: Lifestyle Change Plus Dental Care (LCDC) program improves knowledge, attitude, and practice (KAP) toward oral health and diabetes mellitus among the elderly with type 2 diabetes. J Med Assoc Thai 98: 279-290, 2015.

2. Barrett HL, Dekker Nitert M, Jones L, O'Rourke P, Lust K, Gatford KL, De Blasio MJ, Coat S, Owens JA, Hague WM, et al: Determinants of maternal triglycerides in women with gestational diabetes mellitus in the Metformin in Gestational Diabetes (MiG) study. Diabetes Care 36: 1941-1946, 2013.

3. Jorgensen $\mathrm{CH}$, Gislason $\mathrm{GH}$, Ahlehoff $\mathrm{O}$, Andersson $\mathrm{C}$ Torp-Pedersen C and Hansen PR: Use of secondary prevention pharmacotherapy after first myocardial infarction in patients with diabetes mellitus. BMC Cardiovasc Disord 14: 4, 2014.

4. Harashima SI, Ogura M, Tanaka D, Fukushima T, Wang Y, Koizumi T, Aono M, Murata Y, Seike M and Inagaki N: Sitagliptin add-on to low dosage sulphonylureas: Efficacy and safety of combination therapy on glycaemic control and insulin secretion capacity in type 2 diabetes. Int J Clin Pract 66: 465-476, 2012.

5. Wongchareon W, Phrommintikul A, Kanjanavanit R, Kuanprasert $\mathrm{S}$ and Sukonthasarn A: A predictive model for distinguishing ischemic from non-ischemic cardiomyopathy. J Med Assoc Thai 88: 1689-1696, 2005.

6. Barsotti A, Giannoni A, Di Napoli P and Emdin M: Energy metabolism in the normal and in the diabetic heart. Curr Pharm Des 15: 836-840, 2009.

7. Abu-Sulaiman RM and Subaih B: Congenital heart disease in infants of diabetic mothers: Echocardiographic study. Pediatr Cardiol 25: 137-140, 2004.

8. Mann GE: Nrf2-mediated redox signalling in vascular health and disease. Free Radic Biol Med 75 (Suppl 1): S1, 2014.

9. Wang J, Hu X, Xie J, Xu W and Jiang H: Beta-1-adrenergic receptors mediate Nrf2-HO-1-HMGB1 axis regulation to attenuate hypoxia/reoxygenation-induced cardiomyocytes injury in vitro. Cell Physiol Biochem 35: 767-777, 2015.

10. Jiang G, Liu X, Wang M, Chen H, Chen Z and Qiu T: Oxymatrine ameliorates renal ischemia-reperfusion injury from oxidative stress through Nrf2/HO-1 pathway. Acta Cir Bras 30: 422-429, 2015.

11. Liu J, Wang X, Liu R, Liu Y, Zhang T, Fu H and Hai C: Oleanolic acid co-administration alleviates ethanol-induced hepatic injury via Nrf-2 and ethanol-metabolizing modulating in rats. Chem Biol Interact 221: 88-98, 2014.

12. Xu K, Chu F, Li G, Xu X, Wang P, Song J, Zhou S and Lei H: Oleanolic acid synthetic oligoglycosides: A review on recent progress in biological activities. Pharmazie 69: 483-495, 2014. 
13. Shanmugam MK, Dai X, Kumar AP, Tan BK, Sethi G and Bishayee A: Oleanolic acid and its synthetic derivatives for the prevention and therapy of cancer: Preclinical and clinical evidence. Cancer Lett 346: 206-216, 2014.

14. Mukundwa A, Mukaratirwa S and Masola B: Effects of oleanolic acid on the insulin signaling pathway in skeletal muscle of streptozotocin-induced diabetic male Sprague-Dawley rats. J Diabetes 8: 98-108, 2016.

15. Falcao-Pires I and Leite-Moreira AF: Diabetic cardiomyopathy: Understanding the molecular and cellular basis to progress in diagnosis and treatment. Heart Fail Rev 17: 325-344, 2012.

16. Xie Z, He C and Zou MH: AMP-activated protein kinase modulates cardiac autophagy in diabetic cardiomyopathy. Autophagy 7: 1254-1255, 2011.

17. Cai L, Wang Y, Zhou G, Chen T, Song Y, Li X and Kang YJ: Attenuation by metallothionein of early cardiac cell death via suppression of mitochondrial oxidative stress results in a prevention of diabetic cardiomyopathy. J Am Coll Cardiol 48: 1688-1697, 2006.

18. Kajstura J, Fiordaliso F, Andreoli AM, Li B, Chimenti S, Medow MS, Limana F, Nadal-Ginard B, Leri A and Anversa P: IGF-1 overexpression inhibits the development of diabetic cardiomyopathy and angiotensin II-mediated oxidative stress. Diabetes 50: 1414-1424, 2001.

19. Zhang C, Zhang L, Chen S, Feng B, Lu X, Bai Y, Liang G, Tan Y, Shao M, Skibba M, et al: The prevention of diabetic cardiomyopathy by non-mitogenic acidic fibroblast growth factor is probably mediated by the suppression of oxidative stress and damage. PLoS One 8: e82287, 2013.

20. Tian C, Ouyang X, Lv Q, Zhang Y and Xie W: Cross-talks between microRNAs and mRNAs in pancreatic tissues of streptozotocin-induced type 1 diabetic mice. Biomed Rep 3: 333-342, 2015
21. Badole SL, Jangam GB, Chaudhari SM, Ghule AE and Zanwar AA: L-glutamine supplementation prevents the development of experimental diabetic cardiomyopathy in streptozotocin-nicotinamide induced diabetic rats. PLoS One 9: e92697, 2014

22. Selim ME, Abd-Elhakim YM and Al-Ayadhi LY: Pancreatic response to gold nanoparticles includes decrease of oxidative stress and inflammation in autistic diabetic model. Cell Physiol Biochem 35: 586-600, 2015.

23. Sarkar C, Pal S, Das N and Dinda B: Ameliorative effects of oleanolic acid on fluoride induced metabolic and oxidative dysfunctions in rat brain: Experimental and biochemical studies. Food Chem Toxicol 66: 224-236, 2014.

24. Zeng X, Li J and Li Z: Ginsenoside Rd mitigates myocardial ischemia-reperfusion injury via $\mathrm{Nrf} 2 / \mathrm{HO}-1$ signaling pathway. Int J Clin Exp Med 8: 14497-14504, 2015.

25. Lappalainen J, Lappalainen Z, Oksala NK, Laaksonen DE, Khanna S, Sen CK and Atalay M: Alpha-lipoic acid does not alter stress protein response to acute exercise in diabetic brain. Cell Biochem Funct 28: 644-650, 2010.

26. Hong YA, Lim JH, Kim MY, Kim EN, Koh ES, Shin SJ, Choi BS, Park CW, Chang YS and Chung S: Delayed treatment with oleanolic acid attenuates tubulointerstitial fibrosis in chronic cyclosporine nephropathy through $\mathrm{Nrf} 2 / \mathrm{HO}-1$ signaling. J Transl Med 12: 50, 2014.

27. Yang Y, Zhang J, Liu $\mathrm{H}$ and Zhang L: Change of Nrf2 expression in rat hippocampus in a model of chronic cerebral hypoperfusion. Int J Neurosci 124: 577-584, 2014.

28. Soares MP and Ribeiro AM: Nrf2 as a master regulator of tissue damage control and disease tolerance to infection. Biochem Soc Trans 43: 663-668, 2015. 